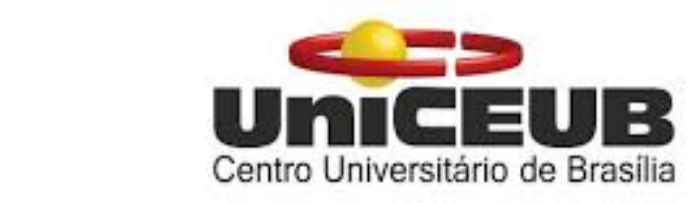

CENTRO UNIVERSITÁRIO DE BRASÍLIA - UniCEUB

PROGRAMA DE INICIAÇÃO CIENTÍFICA

GUSTAVO TEDDE FILHO

INTERNAÇÕES HOSPITALARES E MORTALIDADE EM PACIENTES COM LÚPUS ERITEMATOSO SISTÊMICO NO BRASIL:

UMA ANÁLISE DE 2009 A 2018

BRASÍLIA 


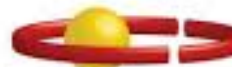 \\ UnICEUB \\ Centro Universitário de Brasilia \\ GUSTAVO TEDDE FILHO
}

\section{INTERNAÇÕES HOSPITALARES E MORTALIDADE EM PACIENTES COM LÚPUS ERITEMATOSO SISTÊMICO NO BRASIL: \\ UMA ANÁLISE DE 2009 A 2018}

\begin{abstract}
Relatório final de Pesquisa de Iniciação
Científica apresentado à Assessoria de Pós-

Graduação e Pesquisa.
\end{abstract}

Orientação: Ana Paula Monteiro Gomides 


\section{AGRADECIMENTOS}

A Deus, por ter me dado sabedoria para enfrentar as dificuldades.

A minha orientadora Ana Paula Monteiro Gomides, pelo suporte, orientações e incentivos no trabalho e durante o curso de Medicina.

A minha família e namorada, por todo carinho e incentivo. 


\section{RESUMO}

O lúpus eritematoso sistêmico é uma doença autoimune, onde ocorre surgimento de autoanticorpos os quais levam a lesões de diversos órgãos e tecidos. As manifestações podem ser de intensidade variável, porém muitas vezes necessitam de internação hospitalar devido a quadros complicados que podem surgir com o decorrer da doença. Na última década houve um significativo aumento das taxas de diagnóstico de lúpus, porém há poucos dados disponíveis a respeito de número de internações mortalidade uma vez que o lúpus eritematoso sistêmico ainda é uma doença subdiagnosticada. Por isso o objetivo geral do artigo é avaliar o número de internações hospitalares e número de óbitos desta população no período de 2009 a 2018. Este é um estudo transversal, descritivo, quantitativo, de natureza documental, com base de dados proveniente do departamento de informática do Sistema Único de Saúde. Os dados resultantes foram submetidos à análise estatística com auxílio do programa Statistical Package for the Social Sciences. No período estudado, foi observado um total de 12.852 internações, sendo $88 \%$ do sexo feminino. A etnia com maior prevalência nas internações foi a parda, com $40 \%$ dos casos. Houve um predomínio de casos em São Paulo, com cerca de 3443 casos, seguido por Minas Gerais, com 1547 casos e Distrito Federal em terceiro lugar, com 1296 casos. São Paulo teve o maior número de óbitos, totalizando 56 óbitos, seguido pelo Rio de Janeiro, com 22 óbitos e em terceiro lugar o Pará, com 20 óbitos. Em relação à mortalidade por etnia, nota-se que, os negros ocupam a primeira posição, pois a cada 100 negros diagnosticados com LES, 2,25 evoluem para óbito. $E$ houve um padrão crescente no número de internações de 2009, com 522 casos, para 2018, com 2312 casos. Dessa forma, o lúpus eritematoso sistêmico é uma doença grave e subdiagnosticada no Brasil, sendo uma importante causa de internações no País. Diante disso, medidas de diagnóstico e tratamento precoces devem ser fortemente estimuladas.

Palavras-chave: Lúpus Eritematoso Sistêmico. Internações hospitalares. Óbitos. 


\section{LISTA DE ABREVIAÇÕES}

LES- Lúpus Eritematoso Sistêmico

SP- São Paulo

MG - Minas Gerais

DF- Distrito Federal

PA- Pará

TO- Tocantins

RR- Roraima

SPSS- Statistical Package for the Social Sciences

EUA - Estados Unidos da América

IAM - Infarto Agudo do Miocárdio 


\section{LISTA DE GRÁFICOS}

Gráfico 1 - Página 8: Número de internações por LES no Brasil de 2009 a 2018.

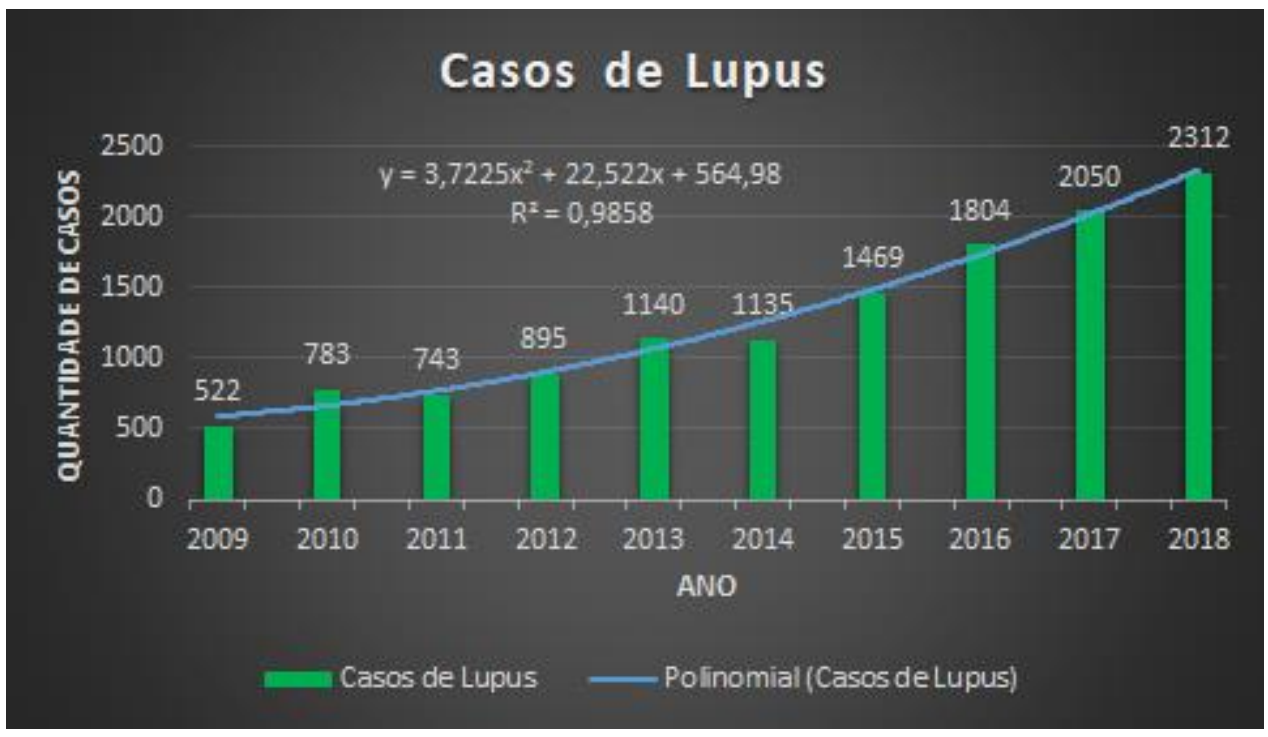

Gráfico 2 - Página 9: Número de internações por raça por LES no Brasil de 2009 a 2018.

\section{Casos de Lupus no comparativo de} raças entre 2009 e 2018 $710 ; 6 \%$

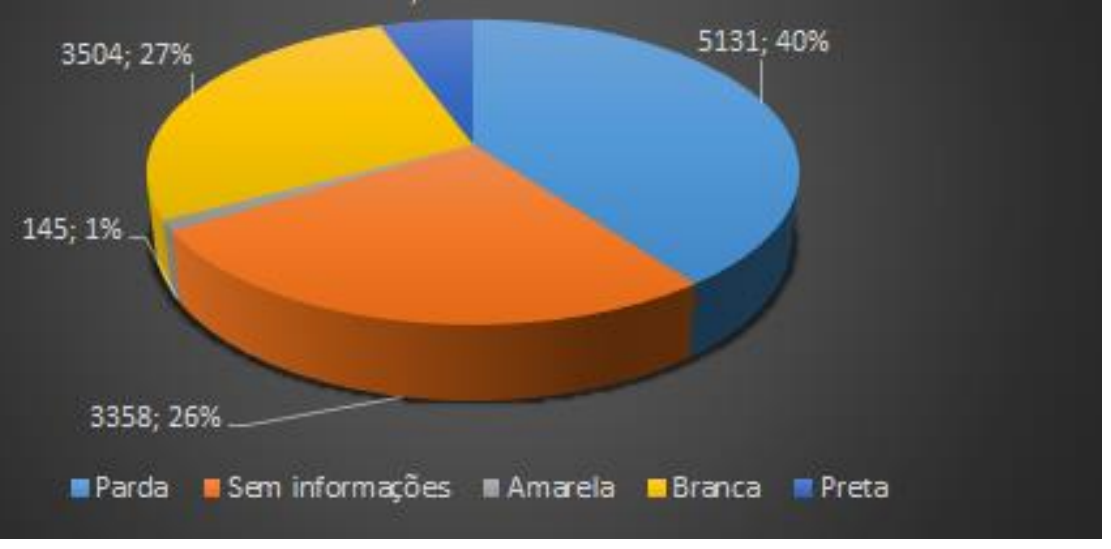


Gráfico 3 - Página 9: Número de internações por unidade federativa por LES no Brasil de 2009 a 2018.

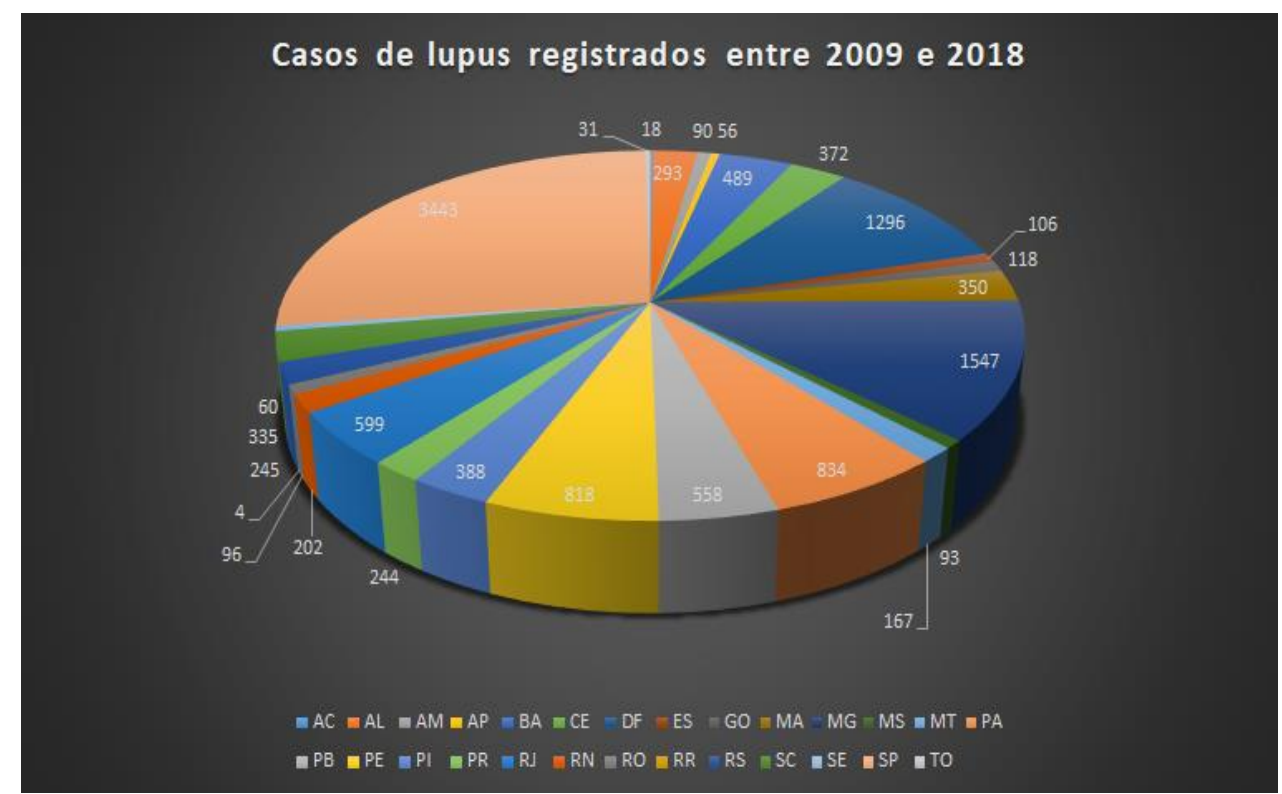

Gráfico 4 - Página 10: Mortalidade por sexo por LES no Brasil de 2009 a 2018.

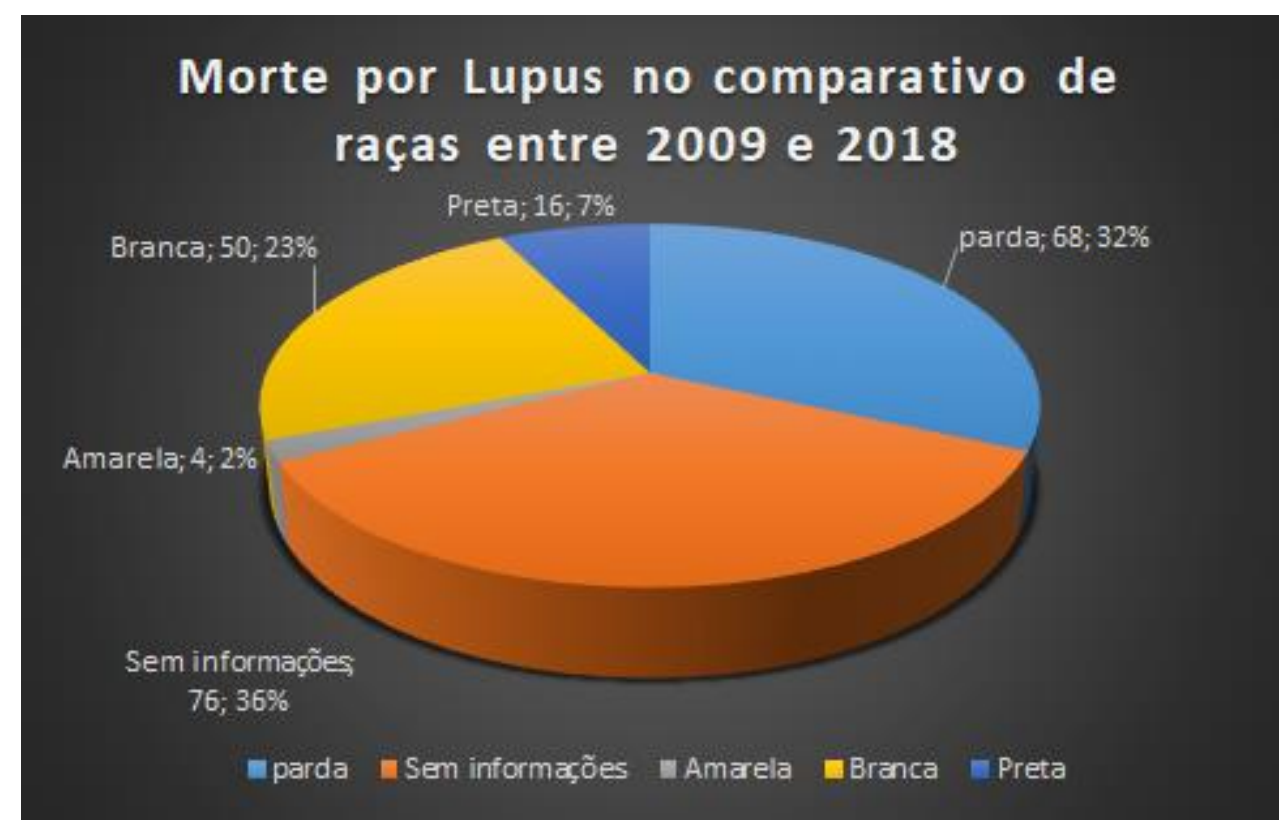


SUMÁRIO

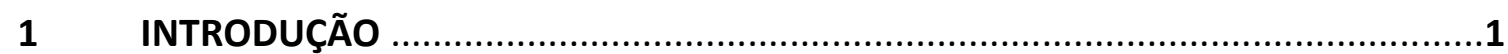

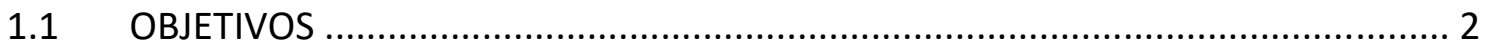

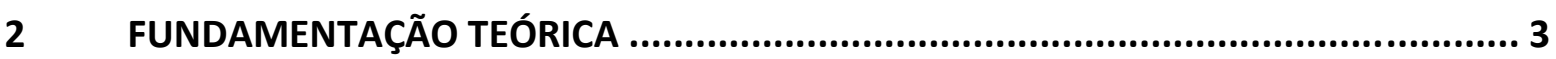

3 MÉTODO

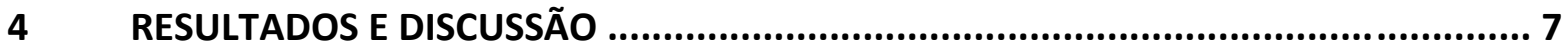

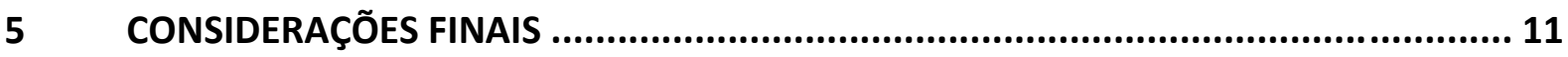

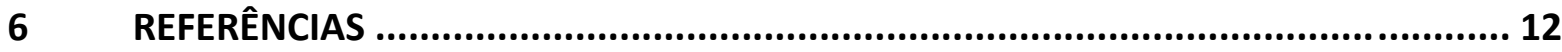




\section{INTRODUÇÃO}

O lúpus eritematoso sistêmico (LES) é uma doença inflamatória, autoimune, caracterizada principalmente pela falha no mecanismo de tolerância e, consequentemente, produção de autoanticorpos que podem causar lesões em múltiplos órgãos e tecidos. ${ }^{1}$ Apesar de ser um tema bastante estudado, sua etiologia ainda não é totalmente esclarecida. Sabe-se da existência de uma correlação com fatores ambientais, genéticos, hormonais, farmacológicos e imunológicos ${ }^{2}$, porém os mecanismos exatos ainda continuam em estudo. As apresentações clínicas do lúpus são extremamente variadas, todavia tendem a acometer predominantemente articulações, pele, sistema circulatório, membranas serosas, rins e cérebro ${ }^{3}$.

Em virtude das potenciais manifestações graves da doença, principalmente renais e do sistema nervoso central, bem como complicações infecciosas, os pacientes com LES muitas vezes necessitam de tratamento a nível hospitalar durante o curso da doença. $\mathrm{Na}$ literatura, poucos trabalhos avaliaram os índices de internação nesta população no Brasil ${ }^{3,4}$. Silva et al. realizaram uma pesquisa de avaliação das internações por Lúpus no estado de Alagoas entre os anos de 2002 e 2007. Foram analisados o predomínio entre sexos, idade, tempo, número, local de internações e óbitos, e foi encontrada concordância dos dados coletados na pesquisa com o padrão descrito na literatura mundial. Cabe ressaltar que neste estudo foram incluídos apenas pacientes de um estado brasileiro. Não há na literatura nenhum trabalho avaliando internações com pacientes com LES em todo o Brasil.

Devido ao seu caráter inflamatório sistêmico, o LES é um grande fator de risco para doenças do sistema cardiovascular. As lesões endoteliais decorrentes da doença são causadas pela liberação de citocinas, que provocam lesão endotelial e propiciam o aparecimento de placas de ateroma e instabilidade de placas pré-formadas, o que eleva o risco de isquemia tecidual, podendo acometer qualquer órgão do corpo ${ }^{3}$. Desta forma os eventos cardiovasculares, como o infarto agudo do miocárdio $(\mathrm{IAM})^{3}$, são as principais causas de morte nesse grupo.

Nas últimas décadas, devido a evolução dos exames laboratoriais e do tratamento, há descrição de redução das taxas de óbito em pacientes com LES em países desenvolvidos. Entretanto, aproximadamente 33\% dos óbitos acontecem em pacientes com menos de 45 anos, o que torna o problema ainda mais relevante ${ }^{3}$. Em nosso país, sabemos que as 
complicações em pacientes lúpicos decorrem principalmente do baixo nível socioeconômico, associado à falta de escolaridade da população. Esses fatores geram um atraso no diagnóstico da doença, sendo descoberta em fases mais tardias e, muitas vezes, com complicações já presentes ${ }^{3}$. Apesar disso, assim como nos dados sobre internações, não dispomos de informações precisas sobre a mortalidade em pacientes com LES no Brasil como um todo.

Diante do impacto ocasionado pela doença, tanto para os pacientes e familiares, bem como relacionados à Saúde Pública, o conhecimento detalhado das causas de internação e taxas de óbitos em pacientes com LES podem fornecer informações importantes para os médicos envolvidos na assistência e aos gestores dos serviços de saúde.

Dentre os autores que mais pesquisam sobre o tema LES, pode-se destacar a pesquisadora Emília Sato. Porém, não há até o momento estudos avaliando o número de internações e óbitos em pacientes com LES no Brasil. O conhecimento destes dados epidemiológicos são fundamentais para fornecer elementos para o adequado manejo destes pacientes na prática clínica.

\section{OBJETIVOS}

\section{Objetivo geral}

Avaliar o número de internações de pacientes com Lúpus Eritematoso Sistêmico no Brasil e o número de óbitos desta população no período de 2009 a 2018, para que se obtenha informações que, até então, foram pouco exploradas.

\section{Objetivos específicos}

1) Avaliar as características socio-demográficas dos pacientes com LES internados no Brasil: sexo, idade, etnia, unidade federativa;

2) Avaliar as características das internações dos pacientes com LES no Brasil, como número de dias e número de óbtos;

3) Avaliar as características socio-demográficas dos pacientes com LES que evoluíram para óbito no Brasil.

\section{FUNDAMENTAÇÃO TEÓRICA}


O Lúpus Eritematoso Sistêmico (LES) foi descrito primeiramente por Paul Ehrlich em 18995. É uma doença inflamatória, crônica, multissistêmica e autoimune. Apesar de sua etiologia permanecer desconhecida, sabe-se que há influência de fatores autoimunes, ambientais, genéticos, hormonais e farmacológicos ${ }^{6,2}$.

O LES é uma doença multigênica, que causa reações de autoimunidade devido a falhas no mecanismo de tolerância imunológica e criação de autoanticorpos, os quais reconhecem células do próprio indivíduo como antígenos ${ }^{7}$. Entre as principais hipóteses etiológicas do LES, encontra-se a deficiência de componentes do sistema de complemento, principalmente o C2 e C4, e uma mutação cromossômica no gene TREX1, associada ao cromossomo $X$. Além das alterações genéticas, o estrogênio tem importante papel na doença, sendo, por esse motivo, mais prevalente em mulheres. $O$ estradiol acopla-se a receptores dos linfócitos $T$ e $B$, gerando respostas imunes prolongadas, o que favorece o quadro inflamatório da doença. Alterações epigenéticas, como a radiação UV, infecções prévias e Vírus Epstein-Barr (EBV) podem servir como gatilho para produção de autoanticorpos ${ }^{8}$.

O LES possui diversas formas de apresentação, variando entre períodos de remissão e exacerbação. Epidemiologicamente, a doença acomete preferencialmente mulheres no menacme, em uma proporção de 9:16. A doença pode ocorrer em todas as raças, porém, é mais frequente em afrodescendentes ${ }^{8}$.

Os autoanticorpos são o motivo das manifestações clínicas, sendo os dois mais específicos o anticorpo Anti-dsDNA e o Anti-Sm. Este último, porém, tem uma sensibilidade baixa, em torno de $25 \%$, enquanto que o Anti-dsDNA tem sensibilidade próxima de $70 \%$. 0 fator anti-nuclear (FAN), apesar de pouco específico, é o mais sensível, pois positiva em 95\% dos casos de LES. Por esse motivo, é usado para rastreio, já que possui um alto valor preditivo negativo. Quando seu resultado é negativo, o diagnóstico de LES é, na maioria das vezes, descartado ${ }^{8}$.

A doença apresenta picos de mortalidade bimodais. Em sua fase inicial, compreendida pelos primeiros dez anos de doença, as principais causas de morte são a própria atividade inflamatória da doença, nefrite lúpica e infecções. Os eventos tromboembólicos estão mais relacionados a períodos mais tardios da doença, mas não exclusivamente a eles. Ressalta-se ainda que o prognóstico da doença seja menos favorável em afrodescendentes e hispano-americanos, pois tendem a evoluir com formas mais graves 
da doença6. O curso da doença é individualizado e não é restrito a uma sequência de manifestações clínicas ${ }^{9}$.

As manifestações clínicas do LES variam e podem acometer qualquer órgão insidiosamente, de forma isolada ou simultânea. Atinge mais frequentemente pele, articulações, células e vasos sanguíneos, membranas serosas, rins, sistema nervoso central (SNC) e sistema nervoso periférico (SNP) ${ }^{10}$.

As manifestações articulares mais comuns são artralgia e artrite. Diferentemente de artrite reumatoide, as artrites de LES são não-erosivas e possuem caráter migratório e reversível. Essas manifestações acometem com maior proporção os punhos, joelhos, ombros e mãos ${ }^{11}$.

O lúpus eritematoso cutâneo é descrito como lesões maculosas ou papulosas, eritematosas, bem definidas, sendo essas regiões áreas fotossensíveis. A apresentação clássica do LES cutâneo é o rash malar também conhecido como "asa de borboleta", e não possui caráter destrutivo ${ }^{12}$. Ademais, o rash malar lúpico tem a importante característica de não acometer o sulco naso-labial, possibilitando sua diferenciação com o rash da dermatomiosite ${ }^{13}$. Com a progressão da doença, essas lesões tornam-se confluentes e formam placas.

Outra forma do lúpus cutâneo é o lúpus cutâneo discoide, em que essas lesões tornam-se crônicas e normalmente, ao regredirem, deixam uma área cicatricial hipocrômica, associado a telangiectasias. Caso o lúpus discoide ocorra em couro cabeludo, a alopecia nessa região específica será permanente, chamada de alopecia cicatricial. Os locais de acometimento por lesões discoides mais comuns são couro cabeludo, pavilhão auditivo, tórax e porção superior dos braços ${ }^{12}$.

O envolvimento renal está relacionado com as maiores taxas de complicações e mortalidade da doença, o que indica pior prognóstico. A insuficiência renal é a causa de morte em $67 \%$ dos pacientes lúpicos e é uma manifestação usual. As alterações renais cursam com componentes nefríticos e nefróticos, como a hematúria microscópica e a proteinúria subnefrótica (maior que 0,5 gramas em 24 horas) ${ }^{14}$. A classificação da nefrite lúpica tem cunho histológico e recomenda-se biópsia para qualquer paciente que apresente clínica de nefrite ${ }^{8}$.

Outro sistema que pode ser acometido é SNP, que pode ocasionar plegias e paralisias de membros. É uma manifestação rara (cerca de $10 \%$ dos pacientes) e esses sintomas 
ocorrem principalmente devido a vasculites, outra manifestação do lúpus, que causa inflamação da vasa nervorum, levando a uma mononeuropatia ${ }^{15,16}$. Outras manifestações comuns do SNP são desordens autonômicas, síndrome de Guillain Barré, polineuropatia, miastenia gravis, entre outros ${ }^{17}$.

O acometimento do SNC no LES tem como manifestação mais comum a disfunção cognitiva, com dificuldade de memória e raciocínio. ${ }^{18}$ Outra característica importante são as cefaleias, que devem ser diferenciadas da enxaqueca e das cefaleias tensionais8. Podem surgir convulsões e psicoses decorrentes das cerebrites ${ }^{18}$. As manifestações focais, como o infarto cerebral, são causadas por oclusão vascular, enquanto as manifestações psíquicas são derivadas de autoanticorpos, principalmente $O$ anticorpo Anti- $P$, associado ao neurolupus, à psicose e à depressão maior ${ }^{17}$.

Os eventos vasculares não tem sua etiologia totalmente esclarecida. Sabe-se que o paciente lúpico tende a ter chances aumentadas de acidentes vasculares cerebrais (AVE) e infarto agudo do miocárdio (IAM). Todavia, não são apenas naquelas pacientes que possuem o anticorpo antifosfolipidio, apesar de este estar mais associado a eventos tromboembólicos ${ }^{19}$. Essas lesões isquêmicas podem ser causadas por lesão inflamatória ou oclusiva por embolização, principalmente derivadas das vegetações assépticas cardíacas, conhecidas como endocardite de Libman-Sacks ${ }^{20,21}$.

As vasculites lúpicas possuem gatilho imunológico, a partir de deposição de imunocomplexos na parede vascular, que liberam citocinas inflamatórias, levando a vasculite. Esse processo pode acontecer em vasos arteriais e venosos de qualquer calibre ${ }^{22}$. Habitualmente, ocorrem em vasos do trato gastrointestinal, causando sintomas inespecíficos, como náuseas, dores leves e diarreias ${ }^{8}$.

A manifestação torácica mais costumeira do LES é a pleurite, em que a dor está presente em $45 \%$ a $60 \%$ dos casos. É possível que a pleurite evolua com um quadro de derrame pleural em $50 \%$ dos pacientes ${ }^{23}$. Como manifestações cardíacas, há pericardite, miocardite e endocardite de Libman-sack ${ }^{8}$.

Pacientes lúpicos frequentemente sentem fadiga e astenia, pois quadros de anemia de doença crônica, normocítica e normocrômica são comuns. Anemia hemolítica imune também é uma causa para redução dos níveis de hemoglobina, porém, é menos comum. A trombocitopenia pode estar presente no LES, porém, costuma não ser tão importante a 
ponto de causar quadros hemorrágicos espontâneos ou pequenos traumas8. A associação da anemia hemolítica com a trombocitopenia é conhecida como síndrome de Evans ${ }^{24}$.

O diagnóstico do LES baseia-se na combinação de dados clínicos e laboratoriais ${ }^{7}$. Recentemente, os critérios de LES foram revisados de acordo com o Systemic Lupus International Collaborating Clinics (SLICC), contando com 17 critérios, em que a presença de quatro ou mais destes é definidora de LES ${ }^{25}$.

Para comprovação dos anticorpos antinucleares, utilizam-se técnicas de análise de proteínas e ácidos nucleicos, podendo ser utilizada a reação imunoenzimática (ELISA), Western blot e imunofluorescência indireta. A hemaglutinação é a técnica mais atual para detecção de anticorpos específicos e possui alta sensibilidade ${ }^{25}$.

O controle e tratamento da doença baseiam-se no uso de corticosteróides, imunossupressores, especialmente a ciclofosfamida, anti-inflamatórios não-hormonais (AINES), antimaláricos, imunossupressores, imunoglobulina endovenosa, anticorpos anti CD20, transplante de medula e, recentemente, Belimumab. Os principais efeitos colaterais desses medicamentos incluem hipertensão arterial, osteoporose, neoplasia, diabetes, falência ovariana e menopausa precoce ${ }^{26}$.

A terapia não farmacológica deve ser um tratamento adjuvante ao medicamentoso, pois proporcionam melhorias na qualidade de vida do paciente. Observou-se que a realização de terapias comportamentais cognitivas geraram redução das manifestações físicas da doença, além de impactarem positivamente a auto estima dos pacientes ${ }^{27}$. Evitar exposição ao sol, fazer uso de filtros solares e prática cautelosa de exercícios físicos são outras formas não farmacológicas coadjuvantes ao tratamento que devem ser implementadas.

Por ser uma doença incurável, o objetivo terapêutico é manter um estado remissivo, prevenir danos orgânicos e efeitos colaterais dos medicamentos e controlar as comorbidades $^{10}$. É interessante notar que com o aumento das pesquisas e do conhecimento em LES, as possibilidades de tratamento proporcionaram uma maior sobrevida aos pacientes ${ }^{27}$.

\section{MÉTODO}

Estudo transversal, descritivo, quantitativo, de natureza documental, com base de dados proveniente do Sistema Único de Saúde (SUS) pelo Departamento e informática do 
Sistema Único de Saúde (DATASUS), que analisou internações com diagnóstico de FR no Brasil no período de 2009 a 2018. As variáveis de interesse (sexo, idade, etnia, unidade federativa, número de dias de internação e taxa de mortalidade) foram selecionadas e retiradas do referido banco de dados do Brasil de 2009 a 2018. Os critérios de inclusão foram as internações com diagnóstico de LES entre os anos de 2009 a 2018, que continham as variáveis de interesse da pesquisa. Os critérios de exclusão foram as internações registradas no banco de dados que não estavam com as variáveis de interesse preenchidas corretamente. Os dados resultantes foram submetidos à análise estatística (medidas descritivas) com auxílio do programa Statistical Package for the Social Sciences (SPSS).

Para realização da pesquisa foi gasto um período de 12 meses. A pesquisa foi realizada pelo aluna do curso de medicina do UniCEUB Gustavo Tedde Filho. A etapa de revisão de literatura teve um período de 3 meses. As etapas de elaboração do projeto e submissão do projeto teve um período de 2 mês. A etapa de coleta de dados teve um período de 2 meses. A análise estatística dos dados teve um período de 2 meses. A elaboração do relatório final e redação do artigo científico teve um período de 3 meses. As fases de coleta de dados e análise estatística da pesquisa foram realizadas pela internet e as demais etapas (discussões com o orientador, análises dos dados, dentre outras) foram realizadas no Campus do UniCEUB - Asa Norte e pela internet.

\section{RESULTADOS E DISCUSSÃO}

Em nosso estudo verificamos um total de 12.952 internações no período de 2009 a 2018. Houve uma progressão do número de pacientes internados no decorrer dos 10 anos, como se pode observar no gráfico 1.

A maior prevalência no número de internação foi do sexo feminino, com $88 \%$ dos casos, contra $12 \%$ do sexo masculino. A média de idade foi de aproximadamente 32 anos. A maioria dos internados por LES foram de etnia parda (40\%). O número de internações por etnia pode ser visto no gráfico 2 .

Quando analisadas as regiões do Brasil, verificamos que o maior número de casos de pacientes internados foi em São Paulo (SP) com cerca de 3.443 casos, seguido por Minas Gerais (MG), com 1547 casos e Distrito Federal (DF), com 1296 casos. Essas três regiões 
compõem aproximadamente 50\% do total de casos de LES no Brasil nos últimos 10 anos. 0 número de internações por unidade federativa pode ser observado no gráfico 3. O tempo médio de internação foi de 9,19 dias (sendo o mínimo de 1 dia e o máximo de 186 dias de internação).

A mortalidade registrada pelo LES foi de 214 dentre os 12852 pacientes internados, o que corresponde a uma taxa de mortalidade de 1,66. Quando comparamos a mortalidade do LES de 2009 a 2018, SP ocupa o primeiro lugar, com 56 óbitos, seguido pelo Rio de Janeiro, com 22 óbitos e em terceiro lugar o Pará, com 20 óbitos. O DF se encontra em quarto lugar com 19 óbitos. MG ocupa a oitava posição no número de mortes por lúpus, com 7 mortes nos últimos 10 anos, empatado com o estado de Alagoas e Santa Catarina. Pode-se notar que apesar de o estado de MG ter um elevado número de pacientes com LES a taxa de mortalidade da região é baixa.

Em relação à mortalidade por etnia, nota-se que, os negros ocupam a primeira posição (a cada 100 negros diagnosticados com LES, 2,25 evoluem para óbito). O percentual comparativo de morte e etnia de brancos, pardos, amarelos é visto no gráfico 4.

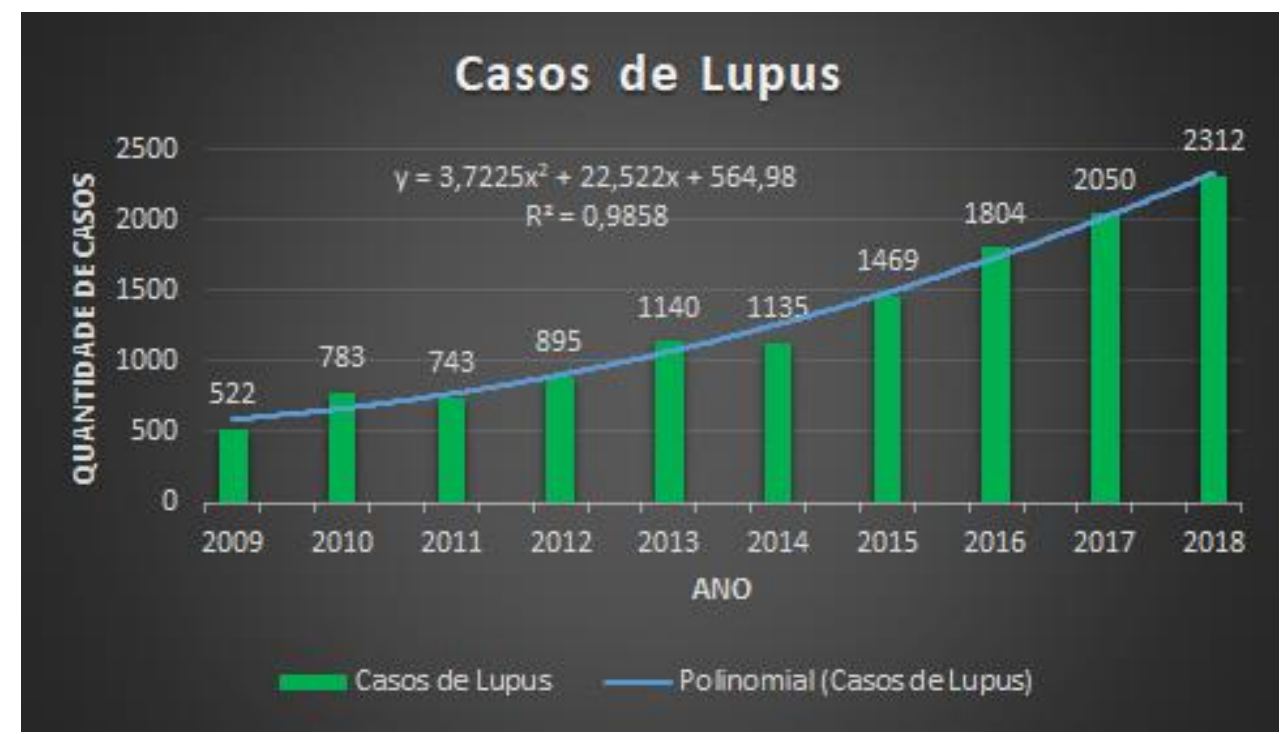

Gráfico 1: Número de internações por LES no Brasil de 2009 a 2018. 


\section{Casos de Lupus no comparativo de} raças entre 2009 e 2018

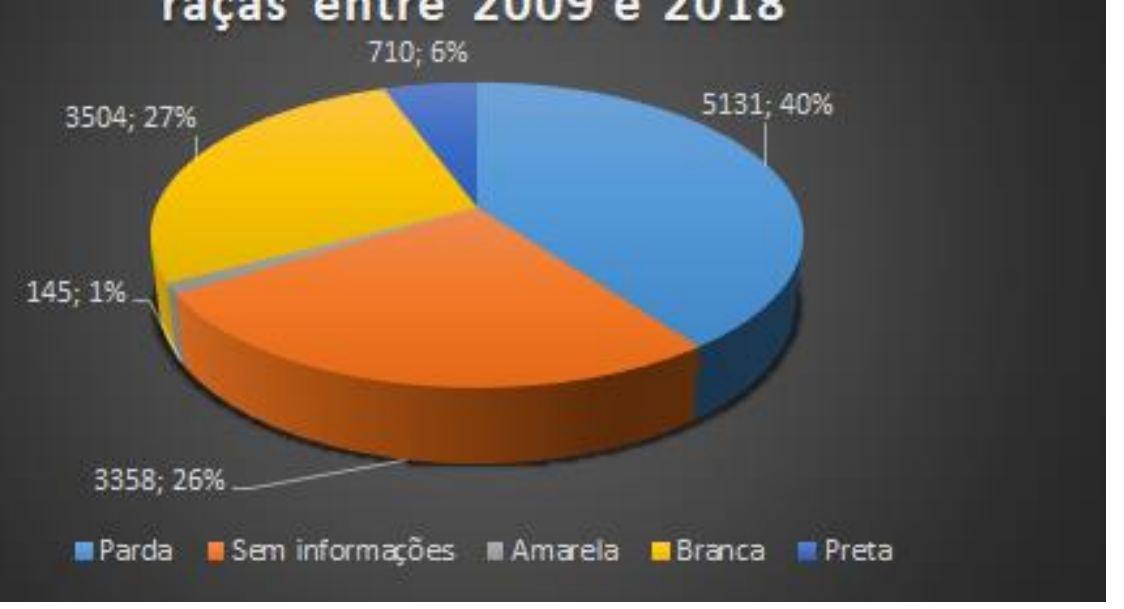

Gráfico 2: Número de internações por raça por LES no Brasil de 2009 a 2018.

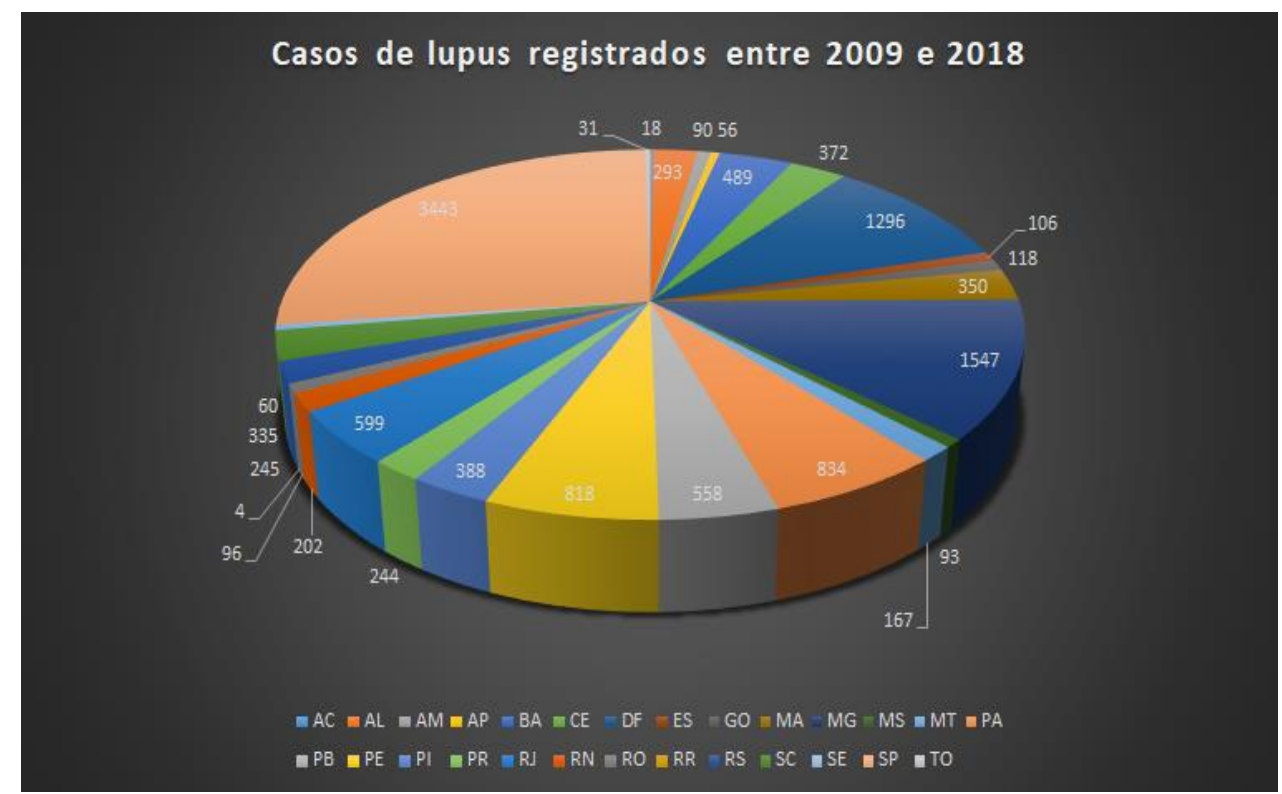

Gráfico 3: Número de internações por unidade federativa por LES no Brasil de 2009 a 2018. 


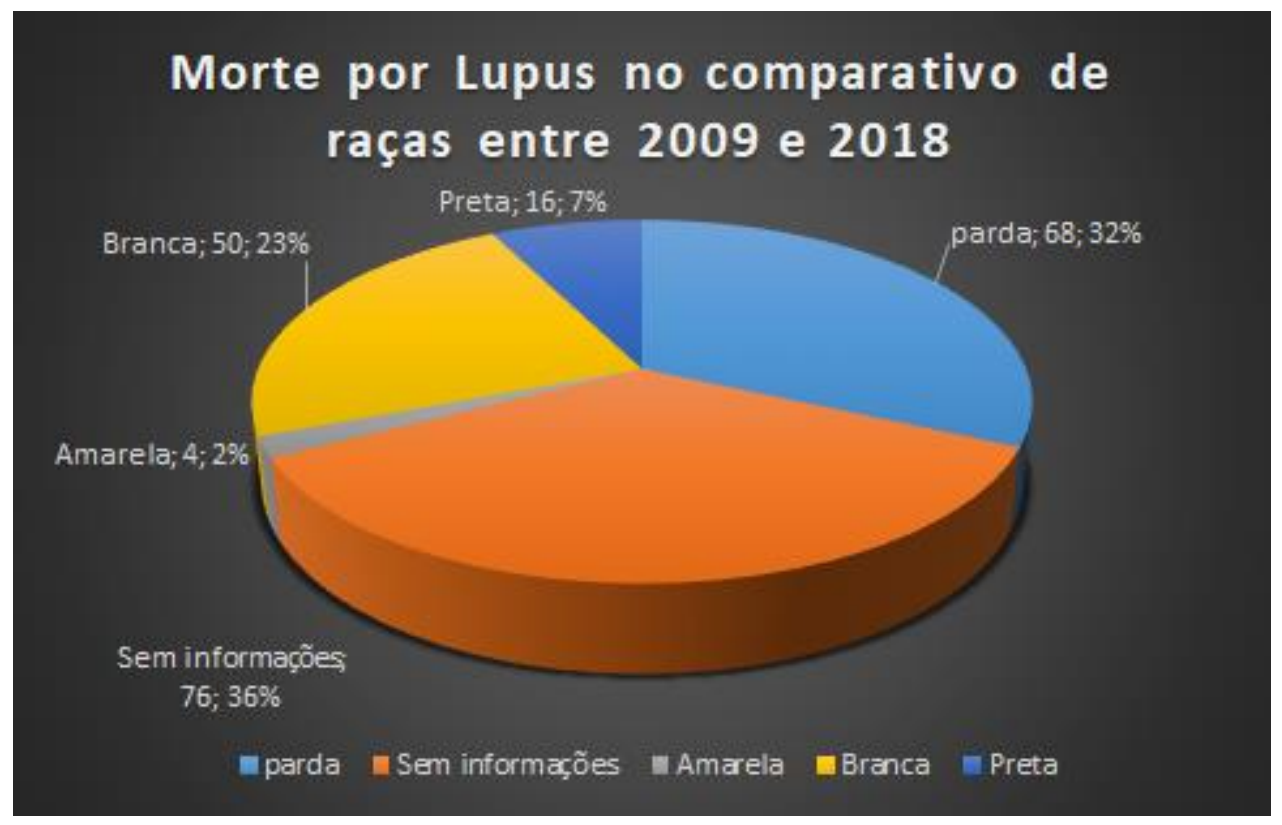

Gráfico 4: Mortalidade por sexo por LES no Brasil de 2009 a 2018.

Observamos que o número de internações foi de 12.852, acompanhado de um padrão crescente do número de internações de 2009 (522 casos) para 2018 (2312 casos). Não encontramos outros trabalhos relacionados à internação por LES e sua distribuição nos últimos anos no Brasil para comparação, mas este fato pode ter ocorrido em virtude do aumento dos diagnósticos fato já observado em outros países. 4

Outro dado percebido em nosso trabalho foi a maior predominância do sexo feminino nos pacientes internados, com idade média de 32 anos. Estes dados têm relação com a incidência da própria doença em todo o mundo, com predomínio em mulheres em idade fértil 9:1 .5,6,7

Também foi observado que a etnia com maior prevalência nas internações de LES é a parda, com 40\% dos casos. Quando comparado com os Estados Unidos da América (EUA), pode-se observar um padrão diferente do encontrado no Brasil, onde existe uma maior prevalência nos afrodescendentes, sendo os caucasianos os menos afetados pela doença. 4

Quanto à distribuição do número de internações por LES nos diferentes estados do Brasil, verificamos que São Paulo, Minas Gerais e o Distrito Federal são responsáveis por aproximadamente, $50 \%$ do total de casos de LES no Brasil nos últimos 10 anos. Não encontramos outros trabalhos publicados sobre o tema para comparação. 
Quando analisada a duração da internação, verificamos que o tempo médio foi de 9,19 dias, semelhante ao tempo médio de internações de LES relatada em outro trabalho realizado no Canadá, com média de 8,5 a 11,2 dias. 7

O presente trabalho apontou que $1,66 \%$ dos internados ao longo dos dez anos, evoluíram para óbito. Entretanto, no ano de 2018 essa taxa de mortalidade aumentou para $1,9 \%$, Esta última taxa é um pouco mais baixa quando comparada a uma grande coorte multicêntrica realizado em 23 centros internacionais e 9.547 pacientes com LES. Neste estudo foi evidenciada uma taxa de mortalidade geral de 2.4 (95\% confidence interval 2.32.5).8 Outro estudo realizado no Brasil avaliou pacientes internados no estado do Alagoas no período de 2002 a 2007 e constatou taxa de mortalidade de $2.4 \%$ nesta população, de forma parecida com nossos resultados.9

Outra questão analisada na nossa pesquisa foi a distribuição do número de óbitos por estado neste período. Percebeu-se que a maioria das mortes por LES se concentram em SP, RJ, PA e DF. Esses estados compõem $54,6 \%$ do total de óbitos. Enquando TO e RR não foi registrada nenhuma morte por LES de 2009-2018. Não encontramos publicações com estas informações no Brasil para comparação.

As principais causas de óbitos no LES relatadas na literatura são as complicações relacionadas à atividade da doença (principalmente renais e no sistema nervoso central), infecções e doença cardiovascular.10,11

No nosso estudo não foi possível determinar a causa exata do óbito em virtude da escassez de informações específicas sobre a doença no banco de dados utilizado.

A falta de informações clínicas detalhadas dos pacientes com LES internados bem como sua evolução são outras limitações deste trabalho. Ainda assim, os dados encontrados são de importante conhecimento para reumatologistas e gestores públicos.

\section{CONSIDERAÇÕES FINAIS}

Esta pesquisa estudou as internações de pacientes com lúpus eritematoso sistêmico no Brasil no período de 2009 a 2018. Avaliou taxa de mortalidade, características sociodemográficas dos pacientes internados (sexo, idade, etnia, unidade federativa), número de dias de internação e número de óbitos. 
A partir dos dados estudados neste trabalho, foi possível observar um elevado número de internações por lúpus eritematoso sistêmico no Brasil no período de 2009 a 2018.

O conhecimento desses dados pode servir como uma forma de alertar quanto à importância do diagnóstico adequado e tratamento precoce de LES. E, dessa maneira, contribuir para redução de complicações crônicas, mortalidade e impacto na saúde individual e coletiva.

\section{REFERÊNCIAS}

1- MOREL, Laurence. Immunometabolism in systemic lupus erythematosus. Nature reviews Rheumatology, v. 13, n. 5, p. 280-290, 2017.

2- DI BATTISTA, Marco et al. One year in review 2018: systemic lupus erythematosus. Clin Exp Rheumatol, v. 36, n. 5, p. 763-777, 2018.

3- MARTINS, Inês Fidalgo. Lupus eritematoso sistémico: uma revisão etiopatogenica. 2016. Dissertação de Mestrado.

4- SILVA, Ana Carolina Santos et al. Internações por Lúpus no estado de Alagoas: 20022007. Revista de Medicina, v. 89, n. 1, p. 43-49, 2010.

5- BENEDEK, Thomas G. History of Lupus. In: Dubois' Lupus Erythematosus and Related Syndromes. Content Repository Only!, 2019. p. 1-14.

6- BORBA, Eduardo Ferreira et al. Consenso de lúpus eritematoso sistêmico. Revista Brasileira de Reumatologia, v. 48, n. 4, p. 196-207, 2008.

7- PIETERSE, Elmar; VAN DER VLAG, Johan. Breaking immunological tolerance in systemic lupus erythematosus. Frontiers in immunology, v. 5, p. 164, $2014 .$.

8- KASPER, Dennis L. et al. Manual de Medicina de Harrison. McGraw Hill Brasil, 2017.

9- PELIÇARI, Karina de Oliveira et al. Manifestações clínicas e laboratoriais associadas a IL-17 no lúpus eritematosos sistêmico juvenil. 2013.

10- RODRIGUES, Douglas Dantas et al. Diagnóstico Clínico E Laboratorial Do Lúpus Eritematoso Sistêmico. Revista de Patologia do Tocantins, v. 4, n. 2, p. 15-20, 2017.

11-RIBEIRO, Daniel Sá et al. Achados de imagem das alterações musculoesqueléticas associadas ao lúpus eritematoso sistêmico. Radiologia Brasileira, v. 44, n. 1, p. 52-58, 2011. 
12- BERBERT, Alceu Luiz Camargo Villela; MANTESE, Sônia Antunes de Oliveira. Lúpus eritematoso cutâneo: aspectos clínicos e laboratoriais. Anais brasileiros de dermatologia, v. 80, n. 2, p. 119-131, 2005.

13- ORTIGOSA, Luciena Cegatto Martins; REIS, Vitor Manoel Silva dos. Dermatomiosite. Anais Brasileiros de Dermatologia, v. 83, n. 3, p. 247-259, 2008.

14- VELOSO, Valéria Soares Pigozzi. Envolvimento renal em pacientes com lúpus eritomatoso sistêmico. 2006.

15- BOUGEA, Anastasia et al. Atualização das manifestações neurológicas das vasculitides e das doenças do tecido conjuntivo: revisão de literatura. Einstein (São Paulo), v. 13, n. 4, p. 627-635, 2015..

16- RADIĆ, Mislav; MARTINOVIĆ KALITERNA, Dušanka; RADIĆ, Josipa. Vascular manifestations of systemic lupus erythematosus. Netherlands journal of medicine, v. $71, n$. 1, p. 10-16, 2013.

17- JOSEPH, Fady G.; SCOLDING, Neil J. Neurolupus. Practical neurology, v. 10, n. 1, p. 4-15, 2010.15

18- ALMEIDA, Sandra et al. Manifestações Neuropsiquiátricas no Lupus Eritematoso Sistémico-A propósito de um caso clínico. Psilogos: Revista do Serviço de Psiquiatria do Hospital Fernando Fonseca, p. 44-52, 2004.

19- GALLI, Monica et al. Lupus anticoagulants are stronger risk factors for thrombosis than anticardiolipin antibodies in the antiphospholipid syndrome: a systematic review of the literature. Blood, v. 101, n. 5, p. 1827-1832, 2003.

20- MOYSSAKIS, loannis et al. Libman-Sacks endocarditis in systemic lupus erythematosus: prevalence, associations, and evolution. The American journal of medicine, v. 120, n. 7, p. 636-642, 2007.

21- FERREIRA, Alexandra Cristina Joaquim. Acidente vascular cerebral associado a endocardite de Libman-Sacks no contexto de sindrome antifosfolipídico e lúpus eritematoso sistémico. 2016. Tese de Doutorado.

22- BACK, Lia Kubelka de Carlos et al. Lúpus Eritematoso Sistêmico: pesquisa de marcadores moleculares de susceptibilidade e prognóstico. 2007.

23- KEANE, Michael P.; LYNCH, Joseph P. Pleuropulmonary manifestations of systemic lupus erythematosus. Thorax, v. 55, n. 2, p. 159-166, 2000.

24- SANTOS, João Pedro Calhau Grade. Síndrome de Evans e lúpus eritematoso sistémico: estado da arte de uma associação incomum. 2017. Tese de Doutorado. 
25- MOCARZEL, Luis Otávio Cardoso et al. Systemic lupus erythematosus: review of cardiovascular aspects. Int J Cardiovasc Sci, v. 28, n. 3, p. 251-61, 2015.

26-SALGADO, J. B. et al. Estudos Econômicos De Belimumabe No Tratamento Do Lúpus Eritematoso Sistêmico-Uma Revisão Sistemática. Value in Health, v. 20, n. 9, p. A893, 2017. 27- OLESIŃSKA, Marzena; SALETRA, Agata. Quality of life in systemic lupus erythematosus and its measurement. Reumatologia, v. 56, n. 1, p. 45, 2018. 